\title{
Preparation and study of the physicochemical properties of psoralen-doxorubicin nanostructured lipid carriers
}

\author{
Tiange Cai MSc${ }^{1}$, Chuling Du MSc${ }^{2}$, Yinghong Huang MSc², Xi Xia MSc² ${ }^{2}$ Yu Cai PhD²
}

T Cai, Y Huang, C Du, X Xia, Y Cai. Preparation and study of the physicochemical properties of psoralen-doxorubicin nanostructured lipid carriers. Curr Res Integr Med 2015;1(3):31-33.

The purpose of the present study was to prepare psoralen-doxorubicin nanostructured lipid carriers (PSO-DOX-NLC) with better physicochemical properties for further study on its multidrug resistance reversal activity on leukemia cells. PSO-DOX-NLC was synthesized by emulsion evaporation and solidification at a low temperature. PSO-DOX-NLC morphology and particle size were examined using transmission electron microscopy, and Zeta potential was determined using photon correlation spectroscopy;

Dsoralen (PSO), an active ingredient with a strong fat-soluble role extracted from legumes, is a type of calcium channel blocker, and can inhibit the P-gp protein pump, assisting chemotherapy drugs with reversing the multidrug resistant effect in cancer cellss $(1,2)$. Doxorubicin (DOX), an anthracycline anticancer drug, whose hydrochloride salt is water-soluble, is primarily used in the treatment of leukemia, lymphoma and breast cancer, having broad-spectrum in antitumour but prone to drug-resistant effects $(3,4)$. The present study was designed to prepare a PSO-DOX-nanostructured lipid carrier (PSO-DOX-NLC) and investigate its physicochemical properties to lay the foundation for further study on its multidrug resistance reversal activity on leukemia cells.

\begin{abstract}
METHODS
Materials

PSO raw material was purchased from Nanjing Spring \& Autumn Biological Engineering Co, Ltd (Nanjing, China [batch number BGZS20140216, content 98\%]. DOX hydrochloride raw material was obtained from Dalian Mellon Biological Technology Co. Ltd (Dlian, China [batch number 20130702, purity $>99 \%$ ]). Glycerylmonostearate (GMS) was provided by Tianjin Bodi Chemical Co, Ltd (Tianjin, Chain). Precirol ATO5, LABRATAC LIPOPHIE WL1349 was acquired from Gattefosse (France). Soya Bean Lecithin of injection grade was produced by Shanghai Tywei Pharmaceutical Co, Ltd (Shanghai, Chain). F68 was obtained from BASF SE (Germany). Tween 80 was purchased from Aladdin Reagent Co, Ltd. Sodium acetate anhydrous was provided by Damao chemical reagent factory (Tianjin, Chain). Glacial acetic acid was acquired from Kemiou Chemical Reagent Co, Ltd (Tianjin, China). Methanol was chromatography grade, water was pure water, and all the other chemicals were analytical grade.
\end{abstract}

\section{Instrumentation}

LC-10AT liquid chromatography; UV-2550 ultraviolet and visible spectrophotometer (Shimadzu, Japan); CP124Celectronic balance (Ohaus, USA); DF-101 Sconstant temperature heating magnetic stirrer (Henan Gongyi Yuhua Instrument Co, Ltd); SB-5200DTD ultrasonic cleaners encapsulation efficiency was also investigated. The results showed that PSO-DOX-NLC possessed a rounded structure, with a mean $( \pm$ SD) particle size of $128.7 \pm 1.8 \mathrm{~nm}$, a polydispersity index of $0.22 \pm 0.01$, a Zeta potential of $20.17 \pm 0.31 \mathrm{mV}$, a psoralen encapsulation rate of $76.56 \%$ and a doxorubicin encapsulation rate of $89.48 \%$. Thus, PSO-DOX-NLC prepared by emulsion evaporation and solidification at a low temperature had good physicochemical properties.

Key Words: Doxorubicin; Nanostructured lipid carriers; Physicochemical property; Psoralen

(Ningbo XinyiUltrasonic Equipment Co, Ltd); TECNAI 10 transmission electron microscope (Philips, Netherlands); Zetasizer Nano ZS90 laser particle size analyzer (Malvern, United Kingdom); 30KD ultrafiltration centrifuge tube (Merck Millipore, Germany).

Preparation of PSO-DOX-NLC

\section{METHODS}

PSO-DOX-NLC was prepared by emulsion evaporation and solidification at a low temperature. Taking solid lipid GMS: ATO5 (1:1) in the formula dosage, $20 \%$ liquid lipid of 1349 , lecithin and PSO, dissolving in $20 \mathrm{~mL}$ absolute ethyl alcohol with $78^{\circ} \mathrm{C}$ water bath, and then obtained the organic phase. While taking a proper amount of DOX and F68 to dissolve in $1 \mathrm{~mL}$ pure water and heating to $78^{\circ} \mathrm{C}$, the inner water phase was separarted, and then taking Tween 80 in formula dosage to dissolve in $20 \mathrm{~mL}$ pure water, the external water phase was obtained. While stirring at $1000 \mathrm{rpm}, 1 \mathrm{~mL}$ internal phase was injected into the stirring organic phase and was stirred for 10 min to form colostrum. The colostrum was injected into the stirring external water phase with same temperature, maintaining stirring and emulsifying to form multiple emulsion. The multiple emulsion was poured into $20 \mathrm{~mL} 4^{\circ} \mathrm{C}$ ice water rapidly, when it volatilized to 5 $\mathrm{mL}$, then solidified in low temperature for $1 \mathrm{~h}$ and filtered through a $0.45 \mu \mathrm{m}$ filtermembrane. Finally the PSO-DOX-NLC was obtained.

\section{Chromatographic conditions}

C18 as chromatographic column $(250 \mathrm{~mm} \times 4.6 \mathrm{~mm}, 5 \mu \mathrm{m})$, methanolsodium acetate buffer ( $\mathrm{pH}$ was adjusted to 3.6 with glacial acetic acid) (65:35) as the mobile phase, $1.0 \mathrm{~mL} / \mathrm{min}$ as the flow rate, $254 \mathrm{~nm}$ as the ultraviolet detection wavelength, $25^{\circ} \mathrm{C}$ as the column temperature.

\section{Specific inspection study}

Adding the proper amount of blank NLC and PSO-DOX NLC to the mobile phase for ultrasonic demulsification and filtering with $0.45 \mu \mathrm{m}$ filtermembrane, then taking PSO solution, DOX solution and the mixed reference solution of PSO-DOX, all in a specific concentration, to sample respectively according to $2: 2: 1$ chromatographic condition.

\footnotetext{
${ }^{1}$ Academy of science, LiaoNing University, Shenyang; ${ }^{2}$ College of Pharmacy, JiNan University, Guangzhou, China

Correspondence: Dr Yu Cai, College of Pharmacy, JiNan University, 601 Huangpu Aveneu West, Guangzhou, Guangdong, China 510632. Telephone 8613119582329, e-mail 944423362@qq.com
} 


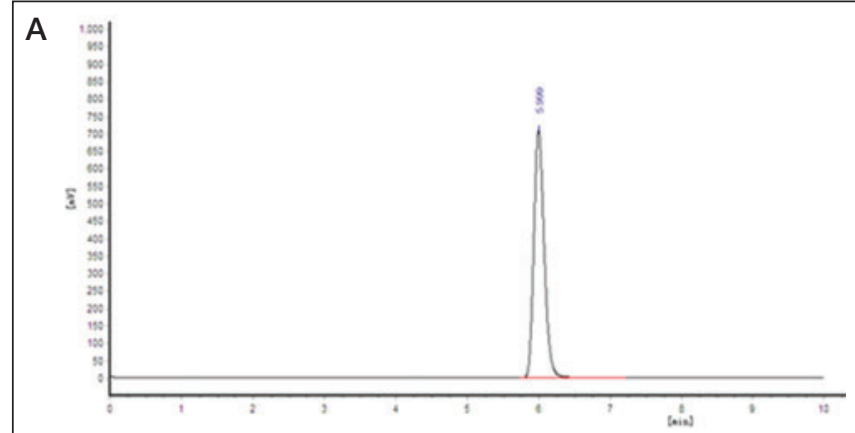

B

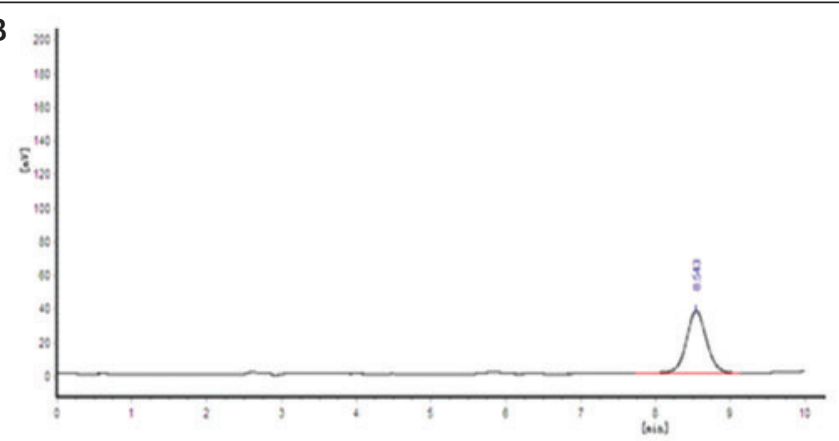

C

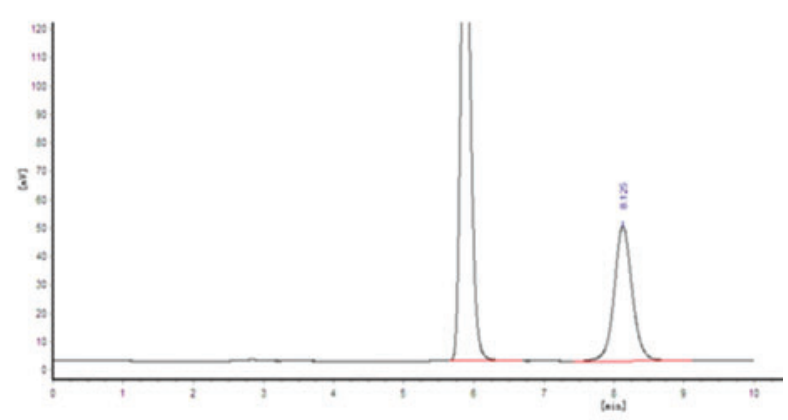

D

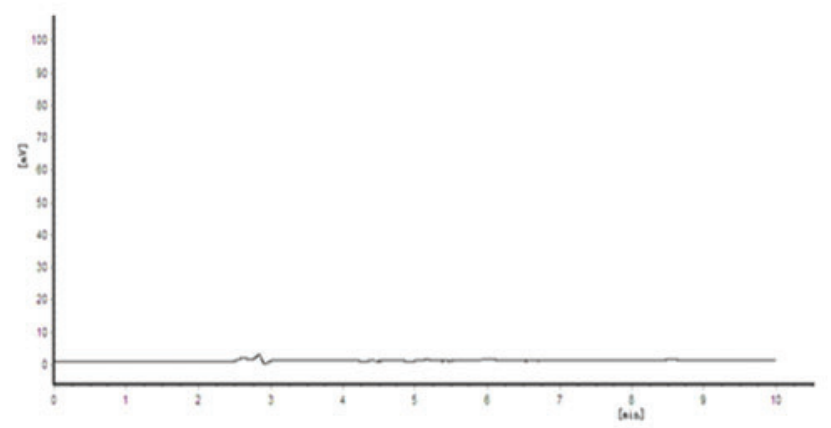

E

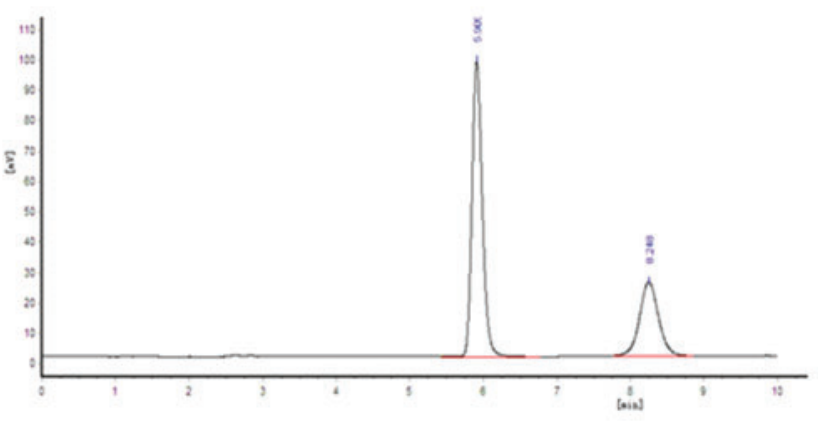

Figure 1) Psoralen (PSO) solution (A); doxorubicin (DOX) solution (B), PSO-DOX mixed reference solution (C), nanolipid carrier (NLC) blank solution (D), PSO-DOX-NLC mixed solution (E)

\section{Linear relationship study}

Precisely adding an appropriate amount of reference solution to $10 \mathrm{~mL}$ volumetric flasks, diluting with methanol to obtain concentrations of 1.0, 2.0, 4.0, 8.0, 16.0 and $32.0 \mu \mathrm{g} / \mathrm{mL}$ mixed reference solution of PSO-DOX. With peak area $(\mathrm{Y})$ as the ordinate, mass concentration $(\mathrm{X}, \mu \mathrm{g} / \mathrm{mL})$ as the abscissa, HPLC was used to investigate the peak area and linear regression was made.

\section{Precision test}

Taking the reference solution of $4 \mu \mathrm{g} / \mathrm{ml}, 16 \mu \mathrm{g} / \mathrm{ml}$ and $32 \mu \mathrm{g} / \mathrm{ml}$ which are in the low, medium and high concentration respectively, investigating their peak area 5 times a day by HPLC in parallel and continuing for 5 days, then calculated the precision RSD.

\section{Recovery test}

Taking $1 \mathrm{~mL}$ blank SLN, adding $0.8 \mathrm{~mL}, 3.2 \mathrm{~mL}$ and $6.4 \mathrm{~mL}$ reference solution into $10 \mathrm{~mL}$ volumetric flasks respectively, diluting with mobile phase to $10 \mathrm{~mL}$, and obtained the low, medium and high concentration samples. Then breaking emulsion via ultrasound, filtering with $0.45 \mu \mathrm{m}$ membrane filtration, investigate their peak area for the average recovery using high-performance liquid chromatography (HPLC), and calculated the ultrafiltration recovery rate.

\section{Stability test}

A reference solution of PSO-DOX ( $8 \mu \mathrm{g} / \mathrm{mL})$, was sampled at $0 \mathrm{~h}, 2 \mathrm{~h}$, $4 \mathrm{~h}, 6 \mathrm{~h}, 12 \mathrm{~h}$ and $24 \mathrm{~h}$, respectively, for calculation of peak area.
Determination method of encapsulation efficiency

The encapsulation efficiency was determined by ultrafiltration in the experiment. Adding an appropriate amount of PSO-DOX-NLC solution into an ultrafiltration centrifuge tube, then investigated the content of free drug $\left(W_{F}\right)$ by HPLC after centrifuging in 12,000 rpm for 20 min. Adding another $1.0 \mathrm{~mL}$ PSO-DOX-NLC solution into the $10 \mathrm{~mL}$ volumetric flask, diluting with the mobile phase to $10 \mathrm{~mL}$, filtering with $0.45 \mu \mathrm{m}$ membrane filter, and investigated the content of total drug $\left(\mathrm{W}_{\mathrm{T}}\right)$ using HPLC. The encapsulation efficiency was then calculated using the following formula:

$$
\mathrm{EE} \%=\frac{W_{\text {total }}-W_{\text {unencapsulated }}}{W_{\text {total }}} \times 100 \%
$$

Investigation of morphology

Putting a proper amount of PSO-DOX-NLC on a copper net with carbon film, and dyeing with $2 \%$ phosphotungstic acid, then observing under transmission electron microscopy after natural drying.

Determination of particle size and electric potential

The PSO-DOX-SLN solution was diluted and its mean particle size and electric potential were determined using a Zeta size nanolaser particle size analyzer (NanoZS90, Malvern Instruments, United Kingdom). 


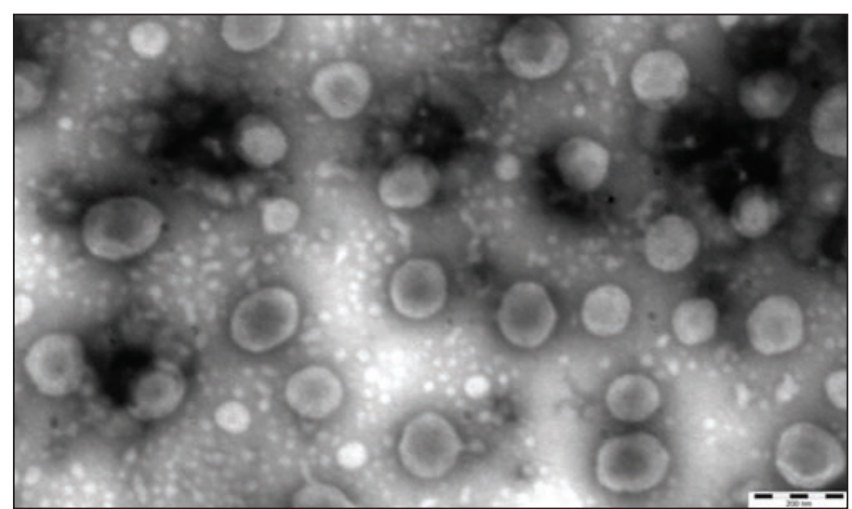

Figure 2) Transmission electron micrograph of psoralen-doxorubicin nanolipid carriers

\section{RESULTS}

Specific inspection study

Under the chromatographic conditions described above, all samples were effectively separated, indicating that the method had high specificity, which is shown in Figure 1.

\section{Linear relationship study}

The standard curve equation of PSO was $\mathrm{Y}=1.1 \times 10^{5} \mathrm{X}+14742$ $(r=0.9998)$ and that of DOX was $Y=54195 X+1877.9 \quad(r=0.9999)$, which suggested that the standard curves for PSO and DOX were in good linear in the concentration ranging from $1 \mu \mathrm{g} / \mathrm{mL}$ to $32 \mu \mathrm{g} / \mathrm{mL}$.

\section{Precision, recovery and stability test}

The results showed that all the RSD of the precision test were under $3 \%$, the recovery of the three concentrations of PSO and DOX were between approximately $95 \%$ and $105 \%$ and $\mathrm{RSD}<3 \%$, The RSD of PSO and DOX were $0.79 \%$ and $1.55 \%$, respectively $(n=6)$, suggesting that the precision, recovery and stability of the method were good.

\section{Morphology of PSO-DOX-NLC}

The morphology of the PSO-DOX-NLC is shown in Figure 2. The particles had a spherical shape, were well dispersed and basically had no adhesion.

\section{The determination of particle size and Zeta potential}

The mean $( \pm \mathrm{SD})$ particle size of the nanoparticles was $128.7 \pm 1.8 \mathrm{~nm}$, PDI was $0.220 .01(n=3)$, and the potential was $(20.17 \pm 0.31) \mathrm{mV}$, as shown in Figure 3.

\section{DISCUSSION}

NLC were prepared by the addition of liquid lipid on the basis of solid lipid nanoparticles. The effect of efflux drug of the solid lipid in the perfect lattice was decreased, and the loading rate and encapsulation efficiency were improved $(5,6)$. In the present experiment, the composite nano structure with high encapsulation efficiency and good physicochemical properties was prepared by emulsion evaporation and solidification at a low temperature, and the method of simultaneous detection of PSO and DOX encapsulation efficiency was established.

Existing research (7) shows that poloxamer with space steric effect, can prevent nanoparticle aggregation. Therefore, in the present experiment, an appropriate amount of F68 was added to the inner water phase and improved the stability of colostrum, while Tween 80 was added to the external water phase to prepare nanoparticles with good appearance and properties. Lecithin, not only a lipid material, but also a type of emulsifier, can improve the stability of nanoparticles. GMS, commonly used as the lipid material, make the nanoparticles have uniform particle size and good dispersion degree and, at the same time, it is a W/O emulsion. Besides, nanoparticles prepared with ATO5 also show good stability and high entrapment efficiency. All experiments simultaneously adopted two types of the solid lipid mentioned above.

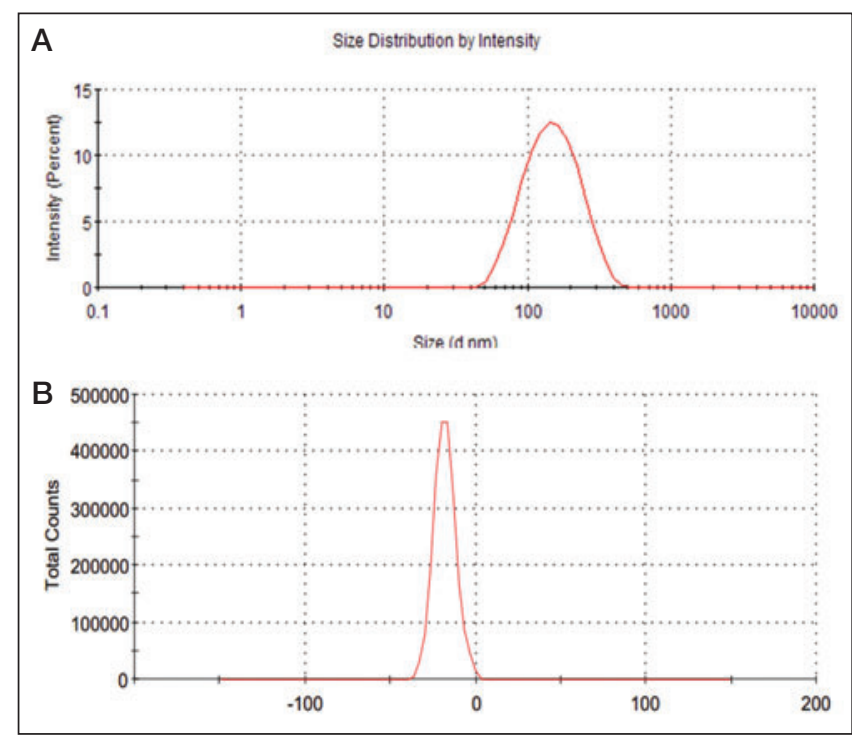

Figure 3) Particle distribution and Zeta-potential of PSO-DOX-NLC

Adriamycin, with a certain water solubility, was dealt with anionic polymer materials (8) and debydrochlorination in the preliminary experiment. However, the stability of the adriamycin nanoparticles prepared this way was inferior to that prepared by multiple emulsion method. Therefore, the multiple emulsion method was chosen.

According to the principle of similar compatility, fat soluble drugs can more easily be prepared into nanoparticles (9). However, the experimental results showed that encapsulation efficiency of adriamycin is superior to that of PSO. The reason may be that adriamycin, a type of cationic drug, tends to combine with lipid materials and adhere to its surface, which has an impact on the release of the drug. The specific mechanism needs further research.

FOUNDATION SUPPORT: The National Natural Science Foundation of China (81273707); Natural Science Foundation of Guangdong (S2013010012880); the Ministry of Education in the New Century Excellent Talents (NECT-12-0677); Science Program of Department of Education of Guangdong (2013KJCX0021); Science and Technology Program of Guangzhou (2014J4500005);

\section{REFERENCES}

1. Tan M, Sun J, Zhao H, et al. The study of psoralen on breast cancer cell line MCF-7 and MDA-MB-231 in vitro. J Beijing University Traditional Chin Med 2009;6:259-362.

2. Cai Y, Chen B, Zhang FH, Zhang RH. The effect of psoralen on the expression of human breast cancer gene Bcl-2. J Toxicol 2005;4:336.

3. Wang HF, Hu Y, Zheng JE, et al. Study on the reverse effect of adriamycin-loaded nanoparticles on K562/DOX cell line.

J Huazhong University Sci Technol (Medical Edition) 2004,33:28992.

4. Wang L, Ke H, Reng DM, et al. The reverse effect of adriamycinloaded nanoparticles on resistant cell lines HL-60/ ADR. Cancer Res Prev Treatment 2008;35:527-32.

5. Huang X, Zhu TT, Luo Q, et al. Study on the preparation and the pharmaceutical properties of Neogambogic acid nanostructured lipid carrier. Chinese Traditonal Drug 2013;44:1400-6.

6. Coo Yoon, Jin Woo Par, In-SooYoon. Solid lipid nanoparticles (SLNs) and nanostructured lipid carriers (NLCs): Recent advances in drug delivery. J Pharm Investig 2013;43:353-62.

7. Li XW, Sun LX, Lin XH, et al. Solid lipid nanoparticles as drug carriers. Prog Chem 2007;19:87-92.

8. Wong HL, Rauth AM, Bendayan R, et al. In vivo evaluation of a new polymer - lipid hybrid nano particle(PLN)for mulation of doxorubicin in a murine solid tumor model. Eur J Pham Biopharm 2007,65:300-8.

9. Xu DH, Gao JQ, Liang WQ, et al. Solid lipid nanoparticles to overcome tumor drug resistance. Chin J Pharm 2010;45:41-2. 